\title{
Material and velocity effects on cavitation erosion pitting
}

\author{
Jean-Pierre Franc ${ }^{\mathrm{a}, *}$, Michel Riondet ${ }^{\mathrm{a}}$, Ayat Karimi ${ }^{\mathrm{b}}$, Georges L. Chahine ${ }^{\mathrm{c}}$ \\ a Grenoble University (LEGI), Grenoble, France \\ b Swiss Federal Institute of Technology (EPFL), Lausanne, Switzerland

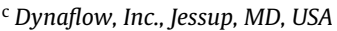

\begin{abstract}
Cavitation erosion during the incubation period was investigated via pitting tests conducted on three different materials: an Aluminum alloy, a Nickel Aluminum Bronze alloy and a Duplex Stainless Steel. Pitting tests were conducted in a cavitation tunnel in the velocity range $45-90 \mathrm{~m} / \mathrm{s}$ at a constant cavitation number. The test section was made of a straight nozzle $16 \mathrm{~mm}$ in diameter discharged into the radial $2.5 \mathrm{~mm}$ space between two flat walls. Cavitation appears in the form of a toroidal cavity attached to the nozzle exit and damage on the samples facing the nozzle is concentrated in a circular ring centered in the cavity closure region. The exposure time was adjusted to avoid pit overlapping. The material surface was examined using a conventional contact profilometer which allowed us to identify the pits, count them, and measure their main characteristics such as depth, surface area, and volume. From these the pitting rate, the coverage rate, and the depth of deformation rate were defined. Pits were classified according to their diameter. For all materials and operating conditions, pitting rate appears to follow an exponential law in relation to the pit diameter. This law depends upon two parameters only, which were identified as the coverage time $\tau$ (i.e. the time required for the surface to be covered by erosion pits) and a characteristic pit diameter $\delta$, which corresponds to the pits whose contribution to the coverage process is the highest. Scaling laws for pitting were derived accounting for both material properties and flow velocity, and a procedure to make pitting test results non-dimensional is proposed. The influence of the material on pitting test results was analyzed. It is shown that the damage is not correlated in simple terms with the elastic limit determined from conventional tensile tests and it is conjectured that other parameters such as the strain rate might play a significant role and should be included in the analysis. The effect of flow velocity on both parameters $\tau$ and $\delta$ was analyzed and a classical power law was found for the influence of the flow velocity on pitting rate for all three materials. Finally, some analysis and discussion is given concerning distributions of pit volume and pit depth.
\end{abstract}

\section{Introduction}

This paper is devoted to the incubation period of the cavitation erosion process which precedes material removal and mass loss. During incubation, damage is characterized by small isolated plastic indentations on the material surface. Each pit is expected to be produced by a cavitation bubble collapsing close to the wall. It is well-known since Lord Rayleigh's work in 1917 that a vapor bubble can actually generate very high loads capable of damaging solid walls when collapsing [1].

Pitting tests have been recognized very early as a helpful technique to estimate the "Cavitation Intensity" as introduced first by Knapp in the 1950s [2,3]. The idea behind pitting tests is to use the material itself as a kind of sensor which will reveal the impact

\footnotetext{
* Corresponding author Tel: +33 4768250 35: fax: +33 476825271

E-mail addresses: Jean-Pierre.Franc@legi.grenoble-inp.fr (J.-P. Franc), ayat.karimi@epfl.ch (A. Karimi), glchahine@dynaflow-inc.com (G.L. Chahine).
}

loads due to bubble collapses, at least the most intense ones. Pitting tests are an alternative to the use of conventional pressure sensors which may be damaged by cavitation and which do not necessarily meet the required conditions for an accurate measurement of the impact loads in terms of rise time and resonant frequency in particular.

Although pitting tests appear as an attractive option to quantify the impact loads, no validated procedure is available yet. Estimating loads from pitting tests requires accurately understanding the material response to the impact load and, by an inverse technique, infer the characteristics of the loads from the measured geometric characteristics of the pits. The high strain rates involved with a pit formation together with the most likely triaxial loading makes this inverse procedure quite difficult. In addition, the material response at the microscopic scale of the collapsing bubble may be affected by multiple parameters such as microstructure, grain size, modes of plastic deformation, etc. The use of pitting tests for the quantification of cavitation intensity is still the subject of investigations and the present work is a contribution to this general objective. 


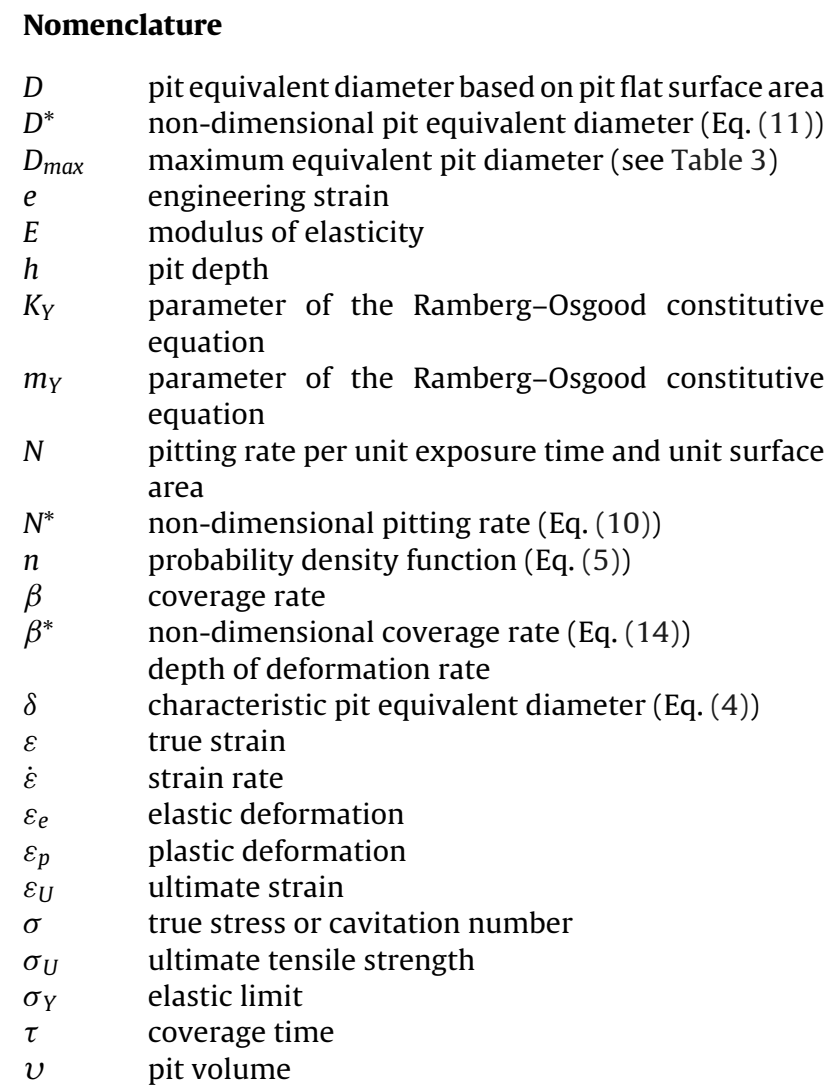

The incubation period and associated pitting has been studied by many investigators using various experimental devices. Cavitation tunnels $[4,5]$ produce cavitation erosion in a flowing liquid whereas vibratory systems [6-12] generate cavitation erosion in an almost stagnant liquid. Other devices have been developed for special purposes such as vortex cavitation generators [13,14], the Hopkinson bar type impact device [15], the magnetic impact testing machine [16], the rotating disk cavitation apparatus $[17,18]$ or submerged cavitating jet devices [19-22]. Some of these techniques were standardized and resulted in American Society for Testing and Materials (ASTM) Standards such as G-32 "Test Method for Cavitation Erosion Using Vibratory Apparatus" and G-134 "Test Method for Erosion of Solid Materials by a Cavitating Liquid Jet". Most of these techniques produce a large spectrum of bubbles of various sizes, at various distances from the eroded sample with possible collective effects which may affect the erosive potential of each individual bubble due to its interaction with the neighboring ones.

Detailed experimental studies have also been made on a single bubble collapsing in a static fluid in order to analyze the basic mechanisms of cavitation damage such as the formation of a high-speed liquid jet and the emission of shock waves. The resulting damage can be much more complex than a unique circular pit because of the formation of the re-entrant jet and a torus like structure which may break up into smaller bubbles whose collapse may cause additional pits of smaller size [23,24].

In the present work, pitting is generated in a cavitation loop. The loop is operated at high enough velocity and pressure in order to be able to investigate cavitation erosion on resistant materials within reasonable exposure times. Because of the large number of bubbles produced simultaneously, the investigation is based on a statistical analysis of erosion pits. Pitting tests have been made on three different materials in order to investigate the influence of the material on pitting results. The influence of the flow velocity

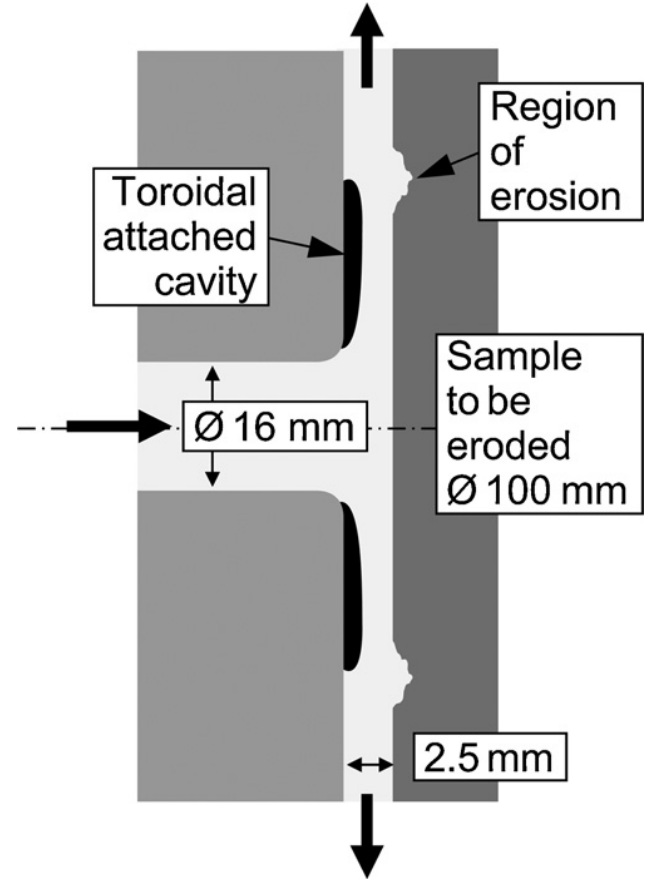

Fig. 1. Sketch of the test section.

is also investigated at constant cavitation number, i.e. for the same cavitation extent in the loop.

This work is part of an ongoing effort between the laboratories of the authors and two US Navy laboratories (NSWCCD and NRL) to derive a procedure for estimating impact loads from pitting tests. The cavitation intensity, which can roughly be defined as the spectrum of impact loads, should obviously be independent of the material used for pitting tests, even though the use of soft materials probably makes it possible to extend the spectra towards loads of small amplitude. The analysis of pitting tests on different materials carried out in this paper is an important step in the development of a technique to assess the cavitation intensity from pitting tests.

\section{Experimental facility and procedures}

\subsection{Test section}

Experiments were conducted in a cavitation flow loop described in details in Ref. [25]. The test section, also described in [25], is axisymmetric and made of a straight nozzle $16 \mathrm{~mm}$ diameter which generates a high velocity flow. As illustrated in Fig. 1, the flow is deflected by the sample to be eroded which is set at a distance of $2.5 \mathrm{~mm}$ from the nozzle exit, and propagates in between two parallel circular plates formed by the plane of the sample and that of the nozzle exit orifice. Cavitation takes the form of a toroidal cavity attached to the nozzle orifice. Cavitation erosion is observed in the closure region of the cavity in the form of a circular ring whose mean diameter is of the order of $50 \mathrm{~mm}$. The radius where maximum damage occurs can easily be determined from mass loss tests and the detection of the radius where the erosion depth is maximum. The flow remains confined in opposition to a cavitating submerged jet.

The maximum operating pressure of the tunnel is 40 bars, which corresponds to a maximum velocity of $90 \mathrm{~m} / \mathrm{s}$. Pitting tests were conducted at different velocities between $45 \mathrm{~m} / \mathrm{s}$ (upstream pressure of 10 bar) and the maximum velocity of $90 \mathrm{~m} / \mathrm{s}$. For all tests, the ambient pressure was adjusted such that when the flow velocity was changed the cavitation number remained constant. This 


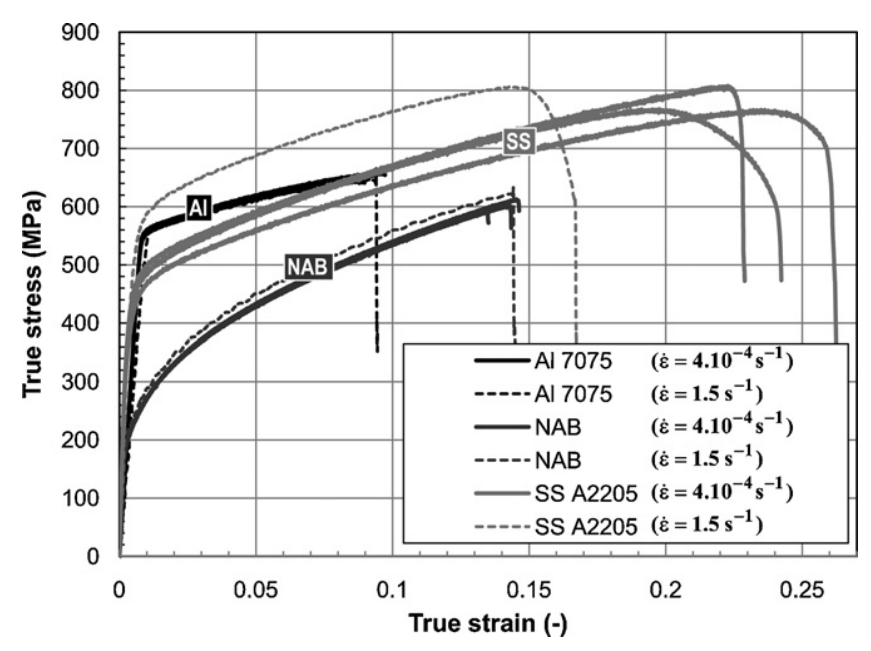

Fig. 2. Stress-strain curves of the three tested materials deduced from conventional tensile tests.

ensured that the cavity length was almost the same for all tests and that a similarity existed between the different flows at different velocities. The velocity effects discussed in this paper are then pure velocity effects which do not include any cavitation number effect.

For each material and each flow velocity, the test duration was adjusted in order to avoid any overlapping of pits which would result in measurement errors. The exposure time was only a few seconds for the tested alloys of Aluminum and Nickel Aluminum Bronze at the maximum velocity and was about $15 \mathrm{~min}$ for the tested Stainless Steel at the smallest velocity. In order to limit the effect of transient flow especially for tests with a short exposure time, a preliminary test was conducted with the purpose of finding out the appropriate settings, i.e. the pressurization level and the operating flowrate. Then, the sample was changed, the pressurization level was adjusted and the pump was started. Flow rate was increased until cavitation inception was identified acoustically. The facility was run at a point just below inception for a sufficient time in order to stabilize the flow. Then, the flow rate was almost instantaneously increased from inception to the final operating point. The increase in flow rate is very rapid and has no effect on the pressurization level. This special procedure minimizes the time needed to reach the operating point. The samples were mechanically polished using a semiautomatic polishing machine (Logitech PM\%) to obtain a metallographic surface of roughness $<0.1 \mu \mathrm{m}$.

\subsection{Materials}

Three materials were selected for comparative tests at LEGI and Dynaflow under this program: Aluminum alloy 7075-T651, Nickel Aluminum Bronze alloy, and Duplex Stainless Steel 2205, named respectively $\mathrm{Al}, \mathrm{NAB}$ and $\mathrm{SS}$ in the present paper. The materials were characterized by conventional tensile tests. Figs. 2 and 3 compare the stress-strain curves of the three materials. Three tests were conducted for each material under quasi-static loads and a small strain rate. Al and NAB show much better reproducibility than SS that might contain more heterogeneities or inclusions.

The stress and strain values presented in Fig. 2 are the true stress $\sigma$ and true strain $\varepsilon$ related to the engineering stress $S$ and engineering strain $e$ by [26-28]:

$\left\{\begin{array}{c}\varepsilon=\ln (1+e) \\ \sigma=s(1+e)\end{array}\right.$

No correction was applied after necking since the diameter reduction and radius change in the necked region were not measured $[26,29]$. Necking was important only for SS.

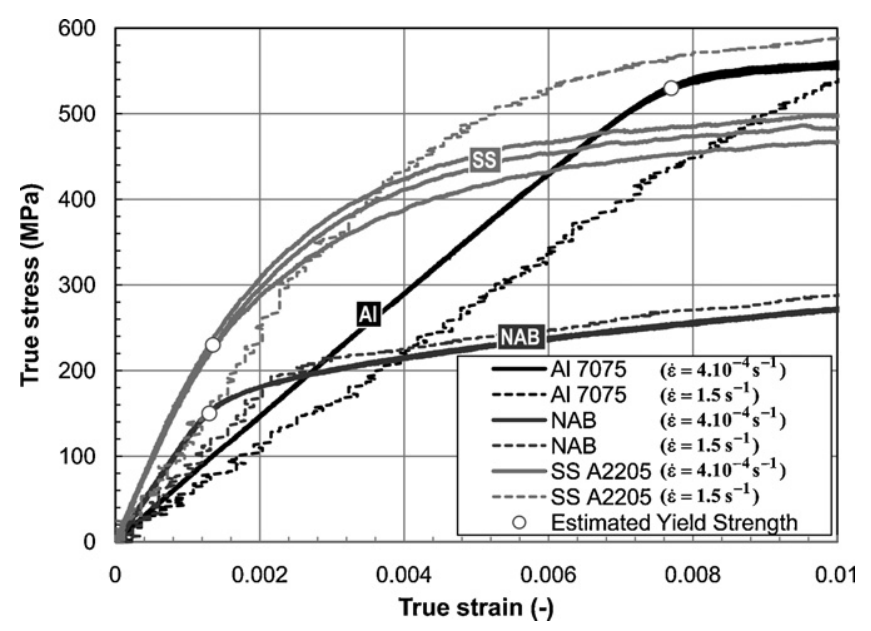

Fig. 3. Stress-strain curves of the three tested materials deduced from conventional tensile tests - enlargement of the elastic domain - the open circles indicate the yield strength given in Table 1 .

The main properties of the tested materials are shown in Table 1. The elastic limit $\sigma_{Y}$ corresponds to the critical stresses where the stress/strain curve $\sigma(\varepsilon)$ departs from the linear elastic relationship:

$\sigma=E \varepsilon_{e}$

where $E$ is the modulus of elasticity. The criterion used for estimating the elastic limit corresponds to a ratio $\varepsilon_{p} / \varepsilon_{e}$ of the permanent plastic deformation $\varepsilon_{p}$ to the elastic deformation $\varepsilon_{e}$ equal to $5 \%$ [30]. A magnification of the elastic region is shown in Fig. 3 together with the elastic limits represented by open circles. The elastic limit which is expected to be the critical stress threshold for the formation of pits will be further considered in Section 5 devoted to the effect of material on pitting.

Table 1 also contains the values of the best fit of parameters $K_{Y}$ and $m_{Y}$ of the Ramberg-Osgood constitutive equation [30]:

$\varepsilon=\varepsilon_{e}+\varepsilon_{p}=\frac{\sigma}{E}+\left[\frac{\sigma-\sigma_{Y}}{K_{Y}}\right]^{m_{Y}}$

Tensile tests have been carried out at a velocity of $30 \mu \mathrm{m} / \mathrm{s}$ which corresponds to a strain rate $\dot{\varepsilon}$ of $4 \times 10^{-4} \mathrm{~s}^{-1}$ considering the length of the samples of $70 \mathrm{~mm}$. However, it is well-known that the characteristic strain rate during the cavitation erosion process is much higher, typically $10^{3} \mathrm{~s}^{-1}$. In order to have indications on the effect of strain rate, tensile tests were also conducted at a higher strain rate of $1.5 \mathrm{~s}^{-1}$. The corresponding results are presented as dashed lines in Figs. 2 and 3. In this range of variation of strain rate, $\mathrm{Al}$ and NAB appear to be only a little sensitive to strain rate. However, the Stainless Steel properties including its elastic limit appear to be significantly affected by the strain rate (see also [31]). These conclusions on the influence of strain rate need to be confirmed by tests at higher strain rate, typically $10^{3} \mathrm{~s}^{-1}$, more representative of the actual strain rate encountered in cavitation erosion.

\subsection{Pit analysis technique}

Various techniques can be found in the literature to analyze pits such as optical profilometry [32,33], laser profilometry [4], scanning electron microscopy [34]. In the present work, a conventional contact profilometer using a stylus with a tip radius of $2 \mu \mathrm{m}$ was used to measure the surface of the sample after pitting. The mesh size for describing the surface was $1 \mu \mathrm{m} \times 1 \mu \mathrm{m}$ and several surfaces of $2 \mathrm{~mm} \times 4 \mathrm{~mm}$ were analyzed (typically 3 ) in order to have a large enough number of pits for the statistical analysis. It has been estimated that a total number of pits between 200 and 300 is generally sufficient [25]. All measurements were made in the region of 
Table 1

Tested materials and main properties.

\begin{tabular}{llll}
\hline Material & Aluminum alloy 7075 T651 (Al) & Nickel Aluminum Bronze alloy (NAB) & Duplex Stainless Steel 2205 (SS) \\
\hline Elastic limit, $\sigma_{Y}$ & $530 \mathrm{MPa}$ & $150 \mathrm{MPa}$ & $230 \mathrm{MPa}$ \\
Ultimate tensile strength, $\sigma_{U}$ & $645-660 \mathrm{MPa}$ & $590-610 \mathrm{MPa}$ & $765-810 \mathrm{MPa}$ \\
Modulus of elasticity, $E$ & $72 \mathrm{GPa}$ & $122 \mathrm{GPa}$ & $186 \mathrm{GPa}$ \\
Ultimate strain, $\varepsilon_{U}$ & $0.086-0.095$ & $0.13-0.15$ & $0.23-0.26$ \\
Ramberg-Osgood parameter, $K_{Y}$ & $447 \mathrm{MPa}$ & $1210 \mathrm{MPa}$ & $910 \mathrm{MPa}$ \\
Ramberg-Osgood parameter, $m_{Y}$ & 1.98 & 2.07 & 3.2 \\
\hline
\end{tabular}

maximum pitting damage, i.e. at the same distance from the center of the sample but at different azimuthal positions.

In order to identify the pits, a cut-off depth was applied during post-processing of the measured surface data. For all results presented in this work (unless otherwise stated), the cut-off depth was chosen at $0.5 \mu \mathrm{m}$ below the original material surface. This choice was considered to give a satisfactory account of the pitted surface for all three materials.

As an example of the influence of the cut-off depth, Fig. 4 shows how the coverage rate is affected by the choice of the threshold. The coverage rate is defined in Section 3.2 and represents the fraction of surface covered by the pits per unit exposure time. The image of the surface where the identified pits have been circled is also shown for three different values of the depth threshold. As expected, the coverage rate (as well as the pitting rate and the depth of deformation rate not presented here), increases when the cut-off depth decreases. In the opinion of the authors, the most serious difficulties when selecting the cut-off depth are to avoid (i) the detection of erroneous pits which would lead to an over-estimation of the pitting rate and (ii) the merger of a pit with neighboring pits or with small surface defects which would alter pit size estimation. This is obviously the case for the smallest cut-off depth of $0.1 \mu \mathrm{m}$ considered in Fig. 4. On the contrary, identification of pits appears to be satisfactory for the two other cases although the shallow pits

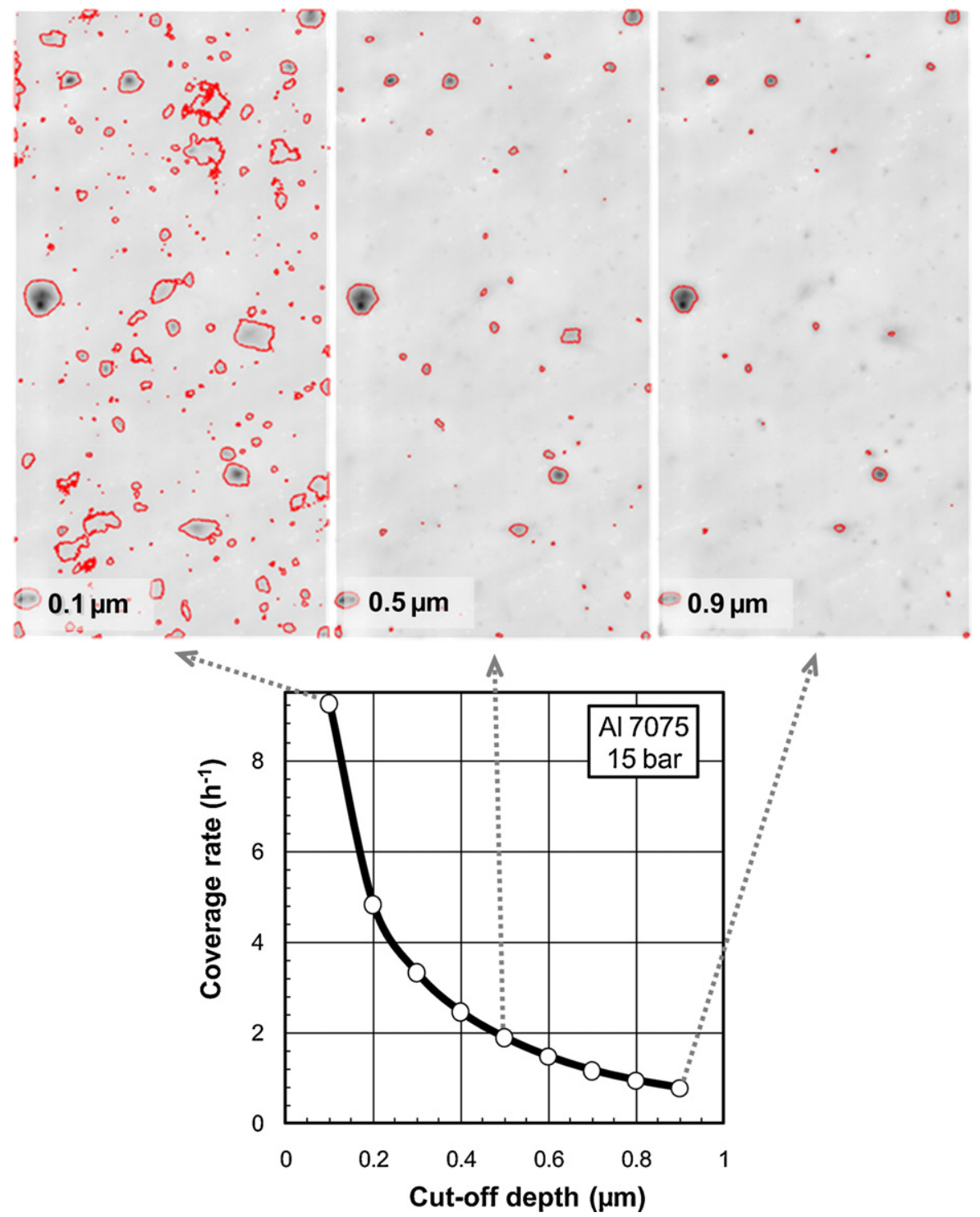

Fig. 4. Influence of cut-off depth on the identified pits and on the coverage rate. The three surfaces presented at the top of the figure are $2 \mathrm{~mm} \times 4 \mathrm{~mm}$. The gray level is an indicator of the local depth. 
are more and more discarded when the cut-off depth increases. The cut-off depth of $0.5 \mu \mathrm{m}$ selected in this work is considered as a good compromise.

\section{Distribution of pits with diameter}

The pit analysis technique used here allows us to identify individual pits and measure their surface areas and volumes. It is then possible to determine the number and the cumulative surface and volume of all the identified pits. It can be expected that pitting data will be proportional to the exposure time as long as no overlapping between pits occurs. However, this requires that the cavitating flow is statistically stable in time and that the material properties remain constant during exposure to cavitation. Both points are discussed below.

Even though the operating conditions are kept constant, cavitating flows are by nature unsteady and involve periodic shedding of bubble clouds. Shedding generally does not affect pitting tests because the exposure time is usually much longer than the shedding period. Therefore, a large number of bubble clouds are shed during the exposure time and the cavitating flow can be considered as stable on average.

Material properties may change over time during exposure to repeated loading because of work hardening and this change could affect the material response during exposure to cavitation. Considering that the plastic zone around a pit is significantly larger than the pit itself, it is important that pits remain sufficiently separated and in any case do not overlap. This guarantees that each new impact falls on a virgin area where the material has not been hardened by a previous impact. If so, no aging effect occurs on the material side and the deformations caused by similar impacts are similar during the exposure to cavitation.

Under such conditions, the number, cumulative surface and cumulative volume of pits can be considered as proportional to the exposure time. In the same way, these data can be considered as proportional to the analyzed surface. This requires mainly that the region of analysis be sufficiently small in comparison to the overall pitted region. If so, the number of pits is constant on equal surface areas and then proportional to the analyzed area. In the present work, we essentially considered the region of maximum damage and did not investigate the variation of pitting damage on both sides of the maximum.

Provided the previous conditions are satisfied, densities can be defined by dividing the measured data by the exposure time and the analyzed surface. This is done for the three data measured here, i.e. the number of pits, their projected surface and their volume, which leads respectively to the pitting rate, the coverage rate and the depth of deformation rate, as indicated in Table 2. Let us observe that each of these quantities can be defined either for all measured pits or, for a more detailed analysis, only for those pits whose diameter is larger than a given value and then become a function of this threshold value. Distribution functions with diameter can then be derived and the contribution of each class of size analyzed.

\subsection{Pitting rate}

The counted pits can be classified according to their diameter. Fig. 5 presents a typical example of the cumulative distribution

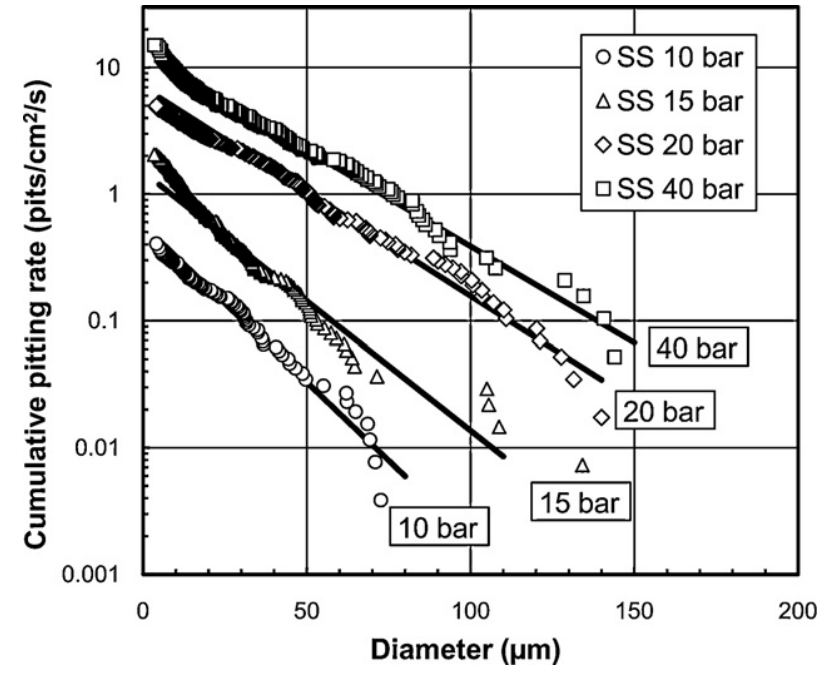

Fig. 5. Cumulative pitting rate as a function of pit diameter for different values of the upstream pressure and a constant cavitation number $\sigma=0.9$ on Duplex Stainless Steel 2205 (cut-off depth: $0.5 \mu \mathrm{m}$ ). Straight lines correspond to an exponential distribution.

function of pitting rate defined as the number $N$ of pits per unit time and unit area counted with a diameter larger than a given value, $D$. The distribution of pitting rate can be approximated, reasonably well, by an exponential law, so that the following mathematical form can be assumed:

$N=\frac{8}{\pi \delta^{2} \tau} e^{-(2 D / \delta)}$

$\delta$ and $\tau$ are two fitting parameters whose physical interpretation will be given later. $\delta$ has a length dimension and $\tau$ has a time dimension so that $N$ has actually the proper dimension of a pitting rate per unit surface area. The factor $8 / \pi$ is introduced to simplify further interpretations.

According to Fig. 5, the approximation by an exponential distribution is not so good for large values of the diameter which correspond to rare events, not necessarily correctly captured during a limited exposure time.

The probability density function $n$ is obtained by differentiation of Eq. (4):

$n=-\frac{d N}{d D}=\frac{16}{\pi \delta^{3} \tau} e^{-(2 D / \delta)}$

This equation gives the number of pits of diameter $D$ per unit bandwidth in diameter. This is also an exponential law where $n$ continuously increases with decreasing diameter, expressing that the number of pits regularly increases when the pit size decreases.

Table 2

Measured and computed data.

\begin{tabular}{|c|c|c|c|}
\hline Measured data & Computed data & Name & Unit \\
\hline $\begin{array}{l}\text { Number of pits } \\
\text { Surface of pits } \\
\text { Volume of pits }\end{array}$ & $\begin{aligned} N & =\frac{\text { number of pits }}{\text { exposure timexanalyzed surface }} \\
\beta & =\frac{\text { surface of pits }}{\text { exposure timexanalyzed surface }} \\
& =\frac{\text { volume of pits }}{\text { exposure timexanalyzed surface }}\end{aligned}$ & $\begin{array}{l}\text { Pitting rate } \\
\text { Coverage rate } \\
\text { Depth of deformation rate }\end{array}$ & $\begin{array}{l}\text { Pits } / \mathrm{m}^{2} / \mathrm{s} \\
\mathrm{s}^{-1} \\
\mathrm{~m} / \mathrm{s}(\text { or } \mu \mathrm{m} / \mathrm{h})\end{array}$ \\
\hline
\end{tabular}




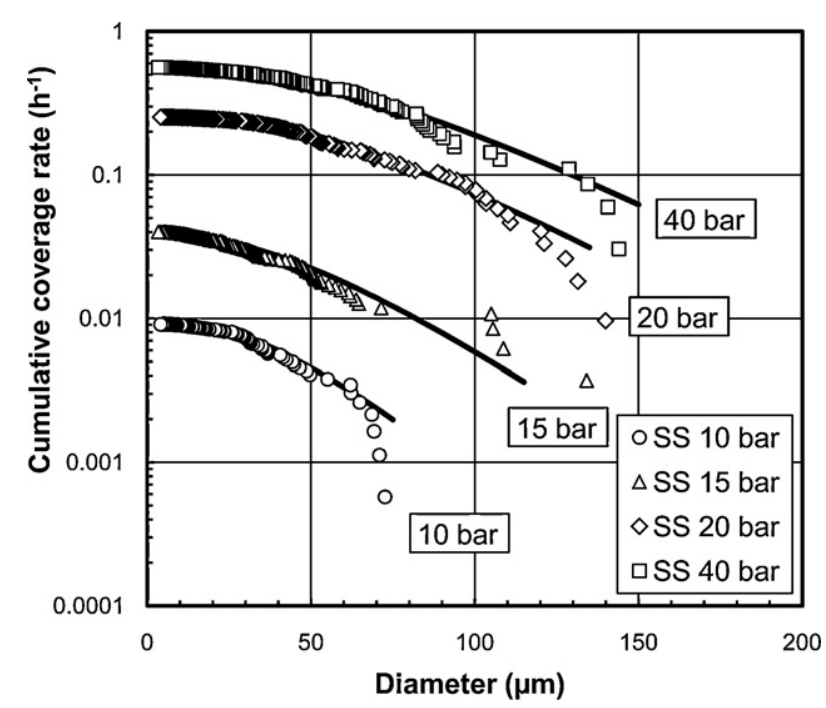

Fig. 6. Cumulative rate of coverage as a function of pit diameter for different values of the upstream pressure and a constant cavitation number $\sigma=0.9$ on Duplex Stainless Steel 2205 (cut-off depth: $0.5 \mu \mathrm{m}$ ). Solid curves correspond to the exponential distribution plotted in Fig. 5.

\subsection{Coverage rate}

It is then possible to integrate the probability density function over the area of all pits of diameter larger than a given value $D$ to obtain the parameter $\beta$ :

$\beta=\int_{D}^{\infty} n \frac{\pi D^{2}}{4} d D=\frac{1}{\tau}\left(1+\frac{2 D}{\delta}+\frac{2 D^{2}}{\delta^{2}}\right) e^{-(2 D / \delta)}$

From a dimensional viewpoint, $\beta$ has the unit of the inverse of time. It can be interpreted as the coverage rate, i.e. the fraction of surface covered by all pits larger than $D$ per unit exposure time to cavitation. Eq. (6) shows that, when $D$ approaches zero, the coverage rate $\beta$ tends to $1 / \tau$. Therefore, the parameter $\tau$ can be interpreted as the time required for the surface to be entirely covered just one time by erosion pits. Note that the factor $8 / \pi$ was introduced in order to remove any multiplication factor in Eq. (6).

The coverage time $\tau$ is expected to play a major role in cavitation erosion, not only in the initial pitting regime but also in the more advanced mass loss regime. The erosion model developed in [25] predicts that (i) the incubation time is proportional to this coverage time and (ii) the erosion rate in the steady state regime of erosion which follows the incubation period, measured by the mean depth of penetration rate (MDPR), is inversely proportional to $\tau$. Even though the incubation time and the erosion rate depend upon several other parameters including material properties, the coverage time $\tau$ appears to be a key parameter which strongly depends upon the flow via the frequency of occurrence of bubble collapse and the characteristic size of the cavitation bubbles.

Let us observe that the so-defined $\tau$ parameter suffers from a certain imprecision since it depends upon the selected cut-off depth used in the pit analysis (see Section 2.3). Moreover, in terms of impact load, it is likely that the load is non uniform on the pit surface. Then, the definition of the pit surface from the cut-off depth without any reference to the impact load distribution over the material needs to be improved. Even though a more precise definition of pit size is needed to derive a more objective parameter, the general concept of coverage time and coverage rate appears to be essential in the physics of cavitation erosion. The concept of coverage can also be found in [35].
The coverage rate is plotted in Fig. 6 in the case of Stainless Steel and for different operating conditions corresponding to different cavitation intensities. The intersection with the $X$-axis, i.e. for $D=0$ corresponds to the total coverage time for all measured pits whatever may be the size, whereas for any non-zero value of $D$, a partial coverage time can be defined corresponding to the contribution of the only pits whose diameter is larger than $D$. This figure confirms the asymptotic behavior of the coverage rate when pit diameter approaches zero, i.e. when all pits are taken into account. This proves that small pits, even though they are the most numerous, do not contribute significantly to the surface coverage.

It appears then that each class of pit diameter does not contribute equally to the coverage process. The probability density function of the coverage rate defined in Eq. (6) is given by:

$-\frac{d \beta}{d D}=\frac{4}{\delta^{3} \tau} D^{2} e^{-(2 D / \delta)}$

This density does not vary in a monotonic way with diameter but presents a maximum, which occurs for a pit diameter equal to $\delta$. This gives a physical interpretation to the second parameter of the exponential distribution law of pitting rate. As a consequence, the major contribution to surface coverage is due to pits whose diameter lies in a bandwidth around $\delta$, skewed towards the larger diameter pits. Larger pits contribute less because of their lower frequency, whereas smaller pits do not contribute appreciably either, because of their small size even though the associated frequency is relatively high. This trend can also be found in [25,33].

Fig. 7 gives typical examples of these probability density functions for the pitting test results presented in Figs. 5 and 6. The characteristic diameter $\delta$ appears to increase with upstream pressure (or equivalently with flow velocity) and our estimates for the present tests on Duplex Stainless Steel 2205 lie in the range $35-57 \mu \mathrm{m}$ according to the flow velocity. The influence of flow velocity will be discussed in more details in Section 6. It is important in practice that pit analysis focuses on this range of diameter which appears to be the most damaging during the incubation period in terms of covered surface. It is conjectured that this range of size is also the most damaging in terms of material failure during the more advanced stages of erosion where mass loss occurs.

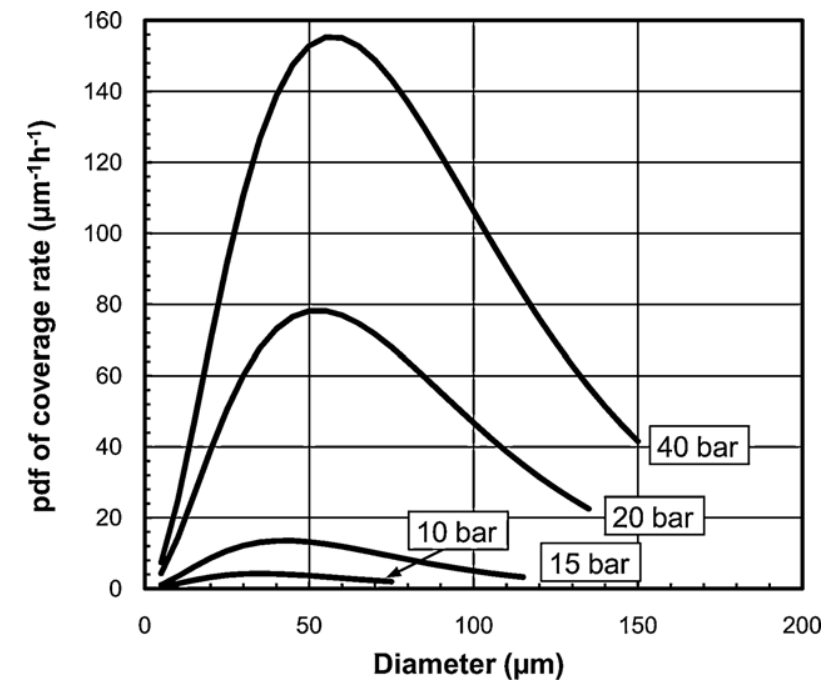

Fig. 7. Estimated probability density function of coverage ratio as a function of pit diameter for different values of the upstream pressure and a constant cavitation number $\sigma=0.9$ on Duplex Stainless Steel 2205 (cut-off depth: $0.5 \mu \mathrm{m}$ ). The curves have been obtained by differentiation of the solid lines plotted in Fig. 6. 


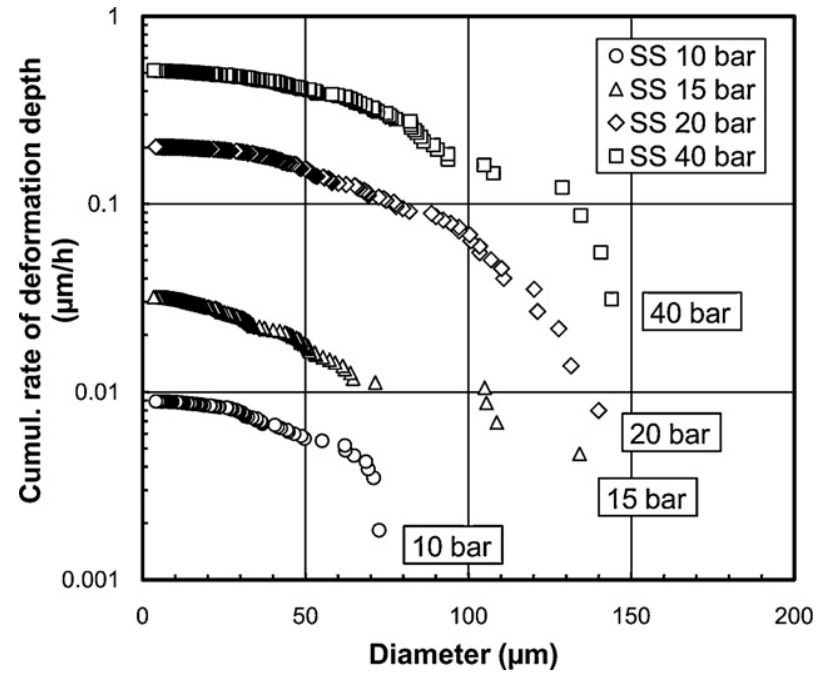

Fig. 8. Cumulative rate of deformation depth as a function of pit diameter for different values of the upstream pressure and a constant cavitation number $\sigma=0.9$ on Duplex Stainless Steel 2205 (cut-off depth: $0.5 \mu \mathrm{m}$ ).

\subsection{Deformation depth rate}

Assuming that a relation between pit volume $v$ and pit diameter is available, it is possible to compute the deformation depth rate, $\gamma$, as a function of pit diameter using the following equation:

$$
=\int_{D}^{\infty} n v d D
$$

As shown in Section 7, pit volume varies like $v \cong 0.47 D^{2.1}$ (cf. Fig. 18). Because of the non-integer value of the exponent, integration of Eq. (6) using the previous equation for $\tau$ is not straightforward and involves a complex Kummer's confluent hypergeometric function. In order to easily extract a general trend, we observe and use the fact that the exponent is actually close to 2 . The rate of deformation depth $\gamma$ should then approach a linear relationship with the coverage rate $\beta$ as it can be concluded from Eqs. (6) and (8) given by $\gamma / \beta \cong 0.6 \mu \mathrm{m}$ if it is assumed that $v \approx 0.47 D^{2}$.

In order to evaluate the validity of this estimate, the ratio $\gamma / \beta$ is plotted as a function of pit diameter in Fig. 9. The figure shows that $\gamma / \beta$ does not vary much and lies mainly between $0.8 \mu \mathrm{m}$ and $1 \mu \mathrm{m}$. The $\gamma / \beta$ values are more scattered for the largest sporadic pits and are no longer almost constant in the case of the lowest flow aggressiveness corresponding to an upstream pressure of 10 bar.

In other words, the behavior of the rate of deformation depth $\gamma$ is generally close to that of the coverage rate $\beta$ and the comparison of Figs. 6 and 8 confirms the similar evolution of both variables with pit diameter.

It was observed previously that there is a class of pit diameters which contributes predominantly to damage in terms of surface. The similar behavior of $\beta$ and $\gamma$ ensures that the same class of pit diameters also contributes primarily to cavitation damage in terms of volume of deformation.

The ratio $\gamma / \beta$ can be interpreted as an average pit depth. The range $0.8-1 \mu \mathrm{m}$ obtained here is consistent with the results presented in Fig. 20.

\section{Scaling laws for pitting tests}

In order to analyze scaling laws applicable to pitting tests, let us consider two different pitting tests. The difference can be either a difference in material or a difference in operating conditions or both

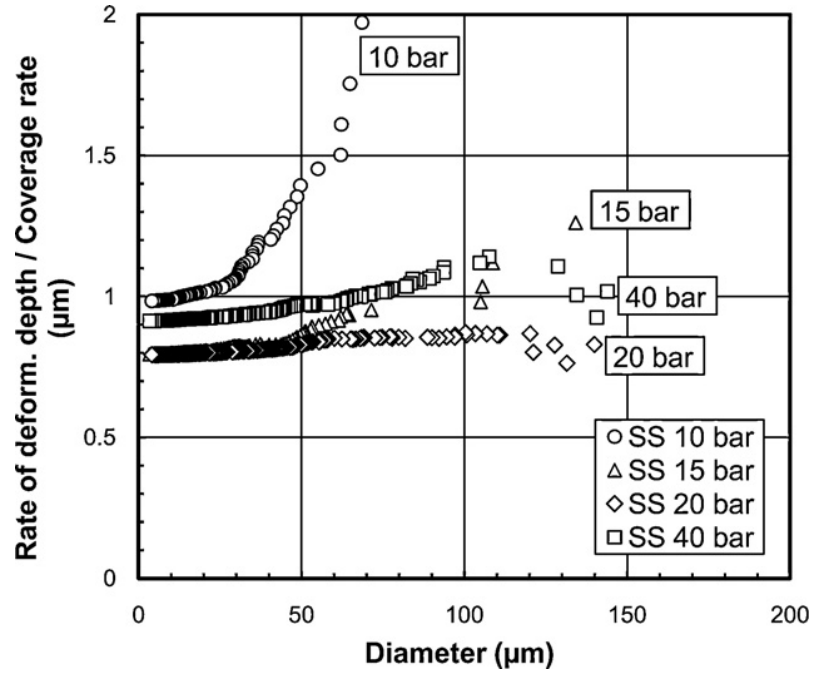

Fig. 9. Ratio of the deformation depth rate $\gamma$ by the coverage rate $\beta$ for different values of the upstream pressure and a constant cavitation number $\sigma=0.9$ on Duplex Stainless Steel 2205 (cut-off depth: $0.5 \mu \mathrm{m}$ ). This ratio can be considered as an estimate of the average pit depth.

of them simultaneously. The two different situations are identified by subscripts 1 and 2 . Pitting rates are written in the form (cf. Eq. (4)):

$$
\begin{aligned}
& N_{1}=\frac{8}{\pi \delta_{1}^{2} \tau_{1}} e^{-\left(2 D / \delta_{1}\right)} \\
& N_{2}=\frac{8}{\pi \delta_{2}^{2} \tau_{2}} e^{-\left(2 D / \delta_{2}\right)}
\end{aligned}
$$

These equations are represented by straight lines in a semi-log graph. Hence, they can be superimposed by a series of two proportional transformations. To transpose pitting test 2 into pitting test 1, Eq. (9) show that diameters have to be multiplied by the ratio $\delta_{2} / \delta_{1}$ and number densities by $\left(\delta_{1} / \delta_{2}\right)^{2}\left(\tau_{1} / \tau_{2}\right)$. Provided that both transformations are applied, the histogram for pitting test 2 reduces to that for pitting test 1 .

Fig. 10 gives an example of this scaling for two different materials, Aluminum alloy and Stainless Steel, and two different operating conditions. It shows that this transformation works well in a large domain of pit size and looses accuracy only for the largest pits whose density suffers from a larger uncertainty due to their rare occurrence.

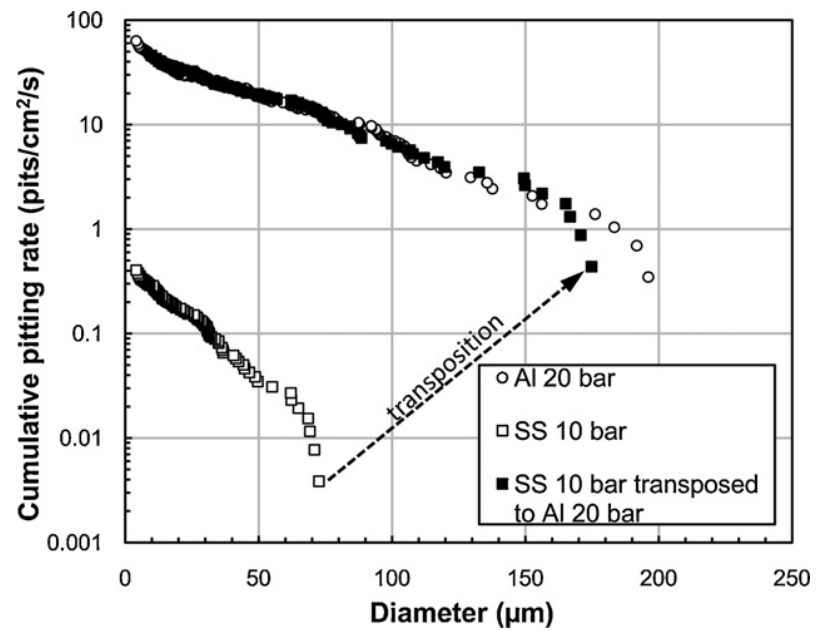

Fig. 10. Principle of the scaling of histograms of pitting rate. Transposition requires two proportional transformations, one on diameters and one on densities. 
This scaling law proves that pit size is scaled like the parameter $\delta$. This parameter is then relevant for characterizing pit size. It will systematically be used below to analyze material and velocity effects on pit size. As for pit density, it is scaled like $\delta^{-2} \tau^{-1}$ as also suggested by a purely dimensional analysis.

In other words, if we introduce the non-dimensional pitting rate defined by:

$N^{*}=\delta^{2} \tau N$

and the non-dimensional diameter defined by:

$D^{*}=\frac{D}{\delta}$

the cumulative histogram of pitting rate takes the following nondimensional form:

$N^{*}=\frac{8}{\pi} e^{-2 D^{*}}$

As for the non-dimensional coverage rate defined by:

$\beta^{*}=\beta \tau$

it is given by the following non-dimensional form of Eq. (6):

$\beta^{*}=\left(1+2 D^{*}+2 D^{* 2}\right) e^{-2 D^{*}}$

with a limit value equal to 1 when diameter approaches zero. These non-dimensional equations give a useful basis to interpret scale effects on pitting tests.

\section{Effect of material properties}

In order to evaluate the influence of material properties, pitting tests were conducted on three different materials (cf. Table 1 ): an Aluminum alloy, a Nickel Aluminum Bronze alloy and a Duplex Stainless Steel.

Figs. 11 and 12 present a comparison of the distributions of cumulative pitting rate and cumulative coverage rate versus diameter for two different operating conditions corresponding to the lowest and the highest speeds. For both cases, the cavitation number was kept constant. (Results on the rate of deformation depth are not presented since they are very similar to those on the coverage rate as explained in Section 3.3.)

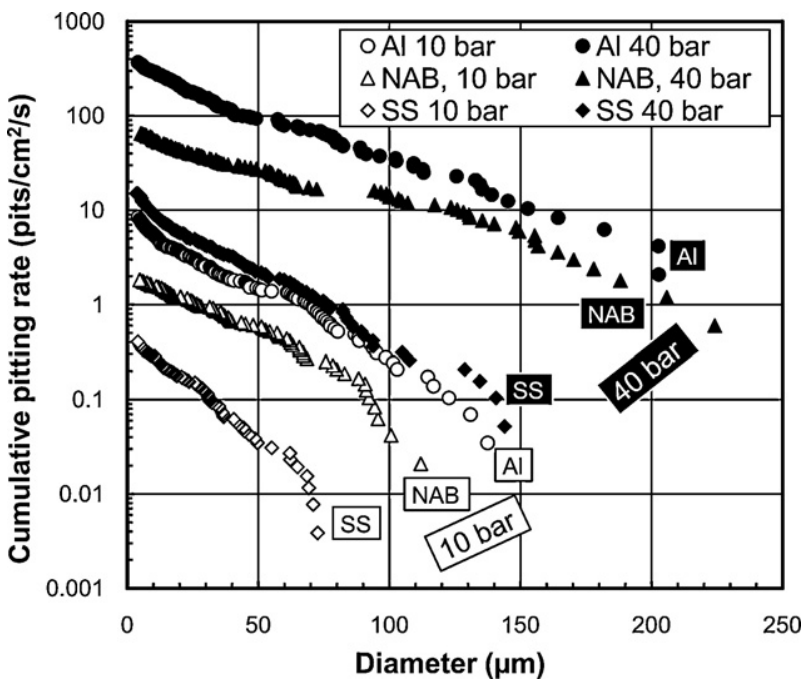

Fig. 11. Comparison of the cumulative pitting rates on three different materials (Aluminum Al 7075, Nickel Aluminum Bronze and Stainless Steel SS2205) for two different values of the upstream pressure (10 bar and 40 bar) and a constant cavitation number $\sigma=0.9$ (cut-off depth: $0.5 \mu \mathrm{m}$ ). Note that the exposure time for pitting test on Aluminum alloy for the most aggressive situation ( $40 \mathrm{bar})$ is very small $(2 \mathrm{~s}$ only).

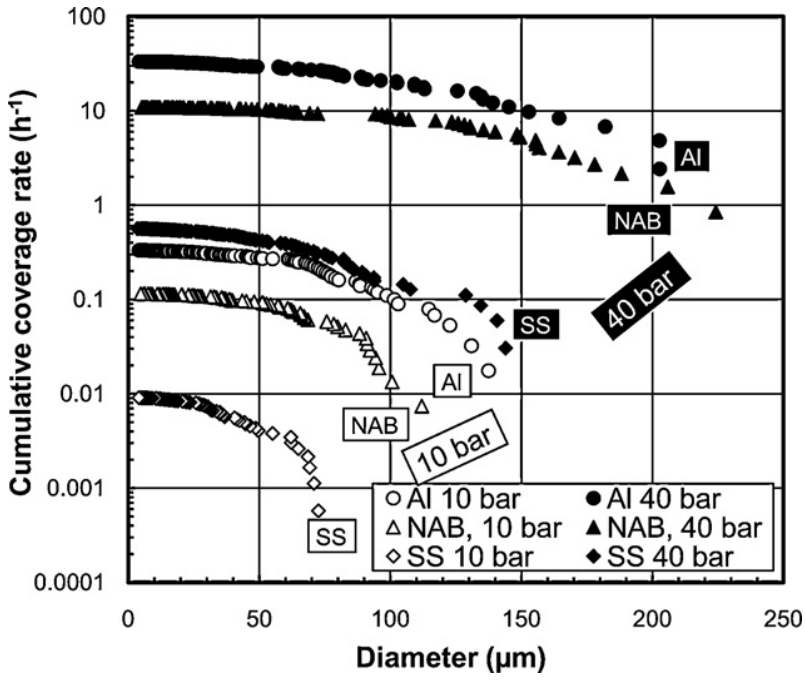

Fig. 12. Comparison of the cumulative coverage rates on three different materials (Aluminum Al7075, Nickel Aluminum Bronze and Stainless Steel SS2205) for two different values of the upstream pressure (10 bar and 40 bar) and a constant cavitation number $\sigma=0.9$ (cut-off depth: $0.5 \mu \mathrm{m}$ ). Note that the exposure time for pitting test on Aluminum alloy for the most aggressive situation ( $40 \mathrm{bar}$ ) is very small ( $2 \mathrm{~s}$ only).

Whatever may be the variable used for characterizing the erosion damage among pitting rate, coverage rate or rate of deformation depth, the most damaged material during the incubation period was Al, followed by NAB and finally SS. It is surprising to observe that $\mathrm{Al}$ was more damaged than SS and NAB whereas its elastic limit evaluated from conventional tensile tests is significantly larger (see Section 2.2). This point is further discussed below.

It is generally assumed that the material has a filtering effect on the histogram of impact loads with a threshold value often considered as the elastic limit [36]. The material is expected to deform elastically when the load is smaller than its elastic limit and to return to its original state after unloading. If the load exceeds the elastic limit, the material is supposed to experience plastic deformation and a pit is formed.

This simple but a priori realistic reasoning should then lead to the smallest pitting rate for $\mathrm{Al}$ as schematically shown in Fig. 13. If the expected ranking of SS and NAB with respect to pitting damage is qualitatively in agreement with experimental data, the prediction concerning $\mathrm{Al}$ does not account for the relatively poor resistance of Al to pitting shown by experiments.

Several reasons can explain this. Firstly, the actual response of the material to cavitation impact loads may be more complex than

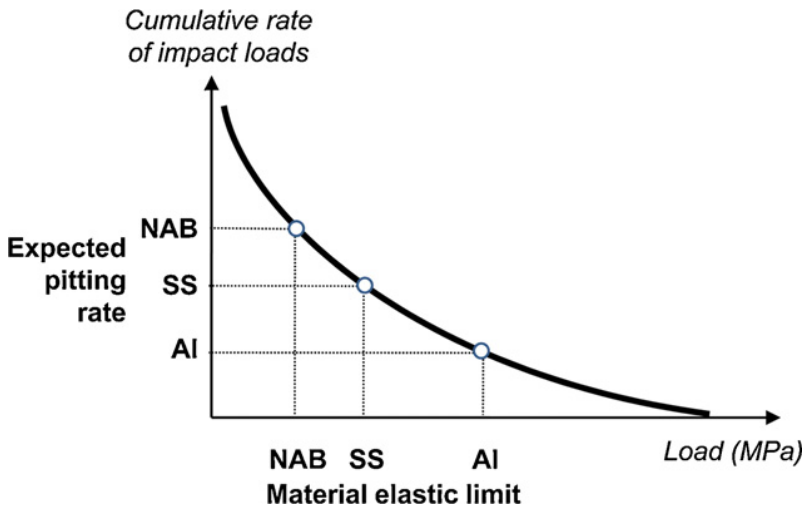

Fig. 13. Schematic illustration of the filtering effect of the load by materials with respect to their elastic limit. 
a simple binary response with the elastic limit as a threshold. It is also possible that the elastic limit is affected by the strain rate. The previous discussion on material ranking was based on the values of the elastic limit determined under quasi-steady conditions by conventional tensile tests. It has been shown in Section 2.2 that the properties of Stainless Steel are strongly affected by the strain rate. Even at the relatively small strain rate of $1.5 \mathrm{~s}^{-1}$, the elastic limit of Stainless Steel is significantly increased and approaches that of Aluminum which is not significantly affected by the strain rate. It can then be expected that the ranking of the three materials with respect to their elastic limit changes with the strain rate, particularly if high strain rates of the order of $10^{3}$ to $10^{4} \mathrm{~s}^{-1}$ are considered. The measurement of material properties at high strain rate using for instance a Hopkinson bar system should allow us to figure out what is the influence of the strain rate. Another advantage of such a test is to provide data under compressive stress analogue to cavitation induced stresses and not under uniaxial tensile stress. It is well known that, unlike an Aluminum alloy, NAB and SS2205 behave differently under tensile and compressive loadings [37]. Finally, let us recall that the influence of the cut-off depth on pit detection remains unclear. The cut-off depth, which has been chosen constant and independent of the material in the present study, might introduce a bias in the pit counting technique since it leads us to discard the shallowest pits.

As explained in Section 4, the factor $\delta_{S S} / \delta_{A l}$ can be used to evaluate the ratio of pit size between Stainless Steel and Aluminum alloy. This ratio together with the similar ratio $\delta_{N A B} / \delta_{A l}$ for NAB is plotted in Fig. 14 as a function of $\mathrm{Al}$ pit size taken as a reference. The characteristic pit size used on the horizontal axis is actually the size parameter $\delta_{A l}$. The different values of $\delta_{A l}$ correspond to different operating conditions. It will be shown in Section 6 that pit size increases with flow velocity so that different values of $\delta_{A l}$ on the horizontal axis correspond to different velocities.

Pit size for NAB appears to be very similar to that for Al with an average value of the ratio of 1.1. Conversely, pits on SS are significantly smaller than on $\mathrm{Al}$ and $\mathrm{NAB}$ and the ratio is about 0.6.

In addition to pitting test results, Fig. 14 presents nanoindentation test results obtained with a spherical indenter of radius $50 \mu \mathrm{m}$. During a nanoindentation test, the load is progressively increased

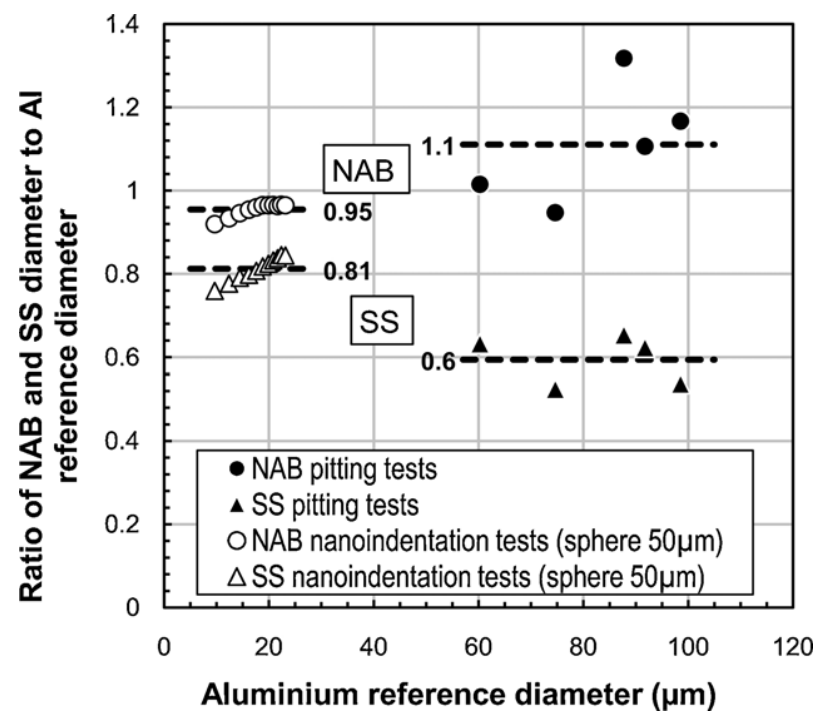

Fig. 14. Comparison of size of indentation (pit or nanoindentation) on Aluminum, Nickel Aluminum Bronze and Stainless Steel. Open symbols correspond to nanoindentation tests with a spherical indenter of $50 \mu \mathrm{m}$ in diameter. Comparisons of nanoindentation diameters are made at equal load. Closed symbols correspond to pitting tests at $10,15,2030$ and 40 bar and a constant cavitation number $\sigma=0.9$ (cut-off depth: $0.5 \mu \mathrm{m}$ )
Table 3

Maximum pit size $D_{\max }$ and ratio of maximum pit size to characteristic pit size $D_{\max } / \delta$.

\begin{tabular}{lllll}
\hline $\begin{array}{l}\text { Operating } \\
\text { conditions }\end{array}$ & Material & $\begin{array}{l}\text { Maximum pit size } \\
\text { measured, } D_{\max }\end{array}$ & $\delta$ & $D_{\max } / \delta$ \\
\hline $40 \mathrm{bar}$ & $\mathrm{Al}$ & $203 \mu \mathrm{m}$ & $88 \mu \mathrm{m}$ & 2.3 \\
& $\mathrm{NAB}$ & $224 \mu \mathrm{m}$ & $116 \mu \mathrm{m}$ & 1.9 \\
& $\mathrm{SS}$ & $144 \mu \mathrm{m}$ & $57 \mu \mathrm{m}$ & 2.5 \\
\multirow{4}{*}{$10 \mathrm{bar}$} & $\mathrm{Al}$ & $137 \mu \mathrm{m}$ & $60 \mu \mathrm{m}$ & 2.3 \\
& $\mathrm{NAB}$ & $112 \mu \mathrm{m}$ & $61 \mu \mathrm{m}$ & 1.8 \\
& $\mathrm{SS}$ & $73 \mu \mathrm{m}$ & $38 \mu \mathrm{m}$ & 1.9 \\
\hline
\end{tabular}

and the diameter of the indentation increases consequently. As for pitting tests, $\mathrm{Al}$ is taken as a reference and the diameter of the indentation on the Aluminum alloy is plotted on the horizontal axis. The different diameters on the $x$-axis correspond to different loads. The same tests are performed on SS and NAB and the diameters of SS and NAB indentations are evaluated at the same loads as Al. Note that the indentation diameter is estimated from the measured penetration depth on the basis of a simple geometric analysis.

The comparison of pitting data and nanoindentation data shows similar qualitative trends. Pit size and indentation size at equal load are smaller for SS than for Al and NAB, which are both comparable. However, nanoindentation tests apparently fail to quantitatively predict the ratio between SS and $\mathrm{Al}$ which is around 0.8 for indentation tests and 0.6 for pitting tests. The analysis of pitting tests using nanoindentation tests needs to be further investigated.

Figs. 11 and 12 show that the maximum pit size significantly depends upon the material. Maximum pit size is difficult to capture because of the small probability of occurrence of the largest pits. Nevertheless, Table 3 presents the diameter of the largest measured pits on the three materials for two different operating conditions and the selected threshold of $0.5 \mu \mathrm{m}$.

The ratio $D_{\max } / \delta$ has an average value of roughly 2 , for all three materials at all operating points. Hence, in spite of the difficulty to accurately determine the maximum pit size $D_{\max }$, it appears that the maximum pit size can reasonably well be predicted by the same scaling law, i.e. varies approximately like the $\delta$ parameter and more precisely like $2 \delta$.

\section{Effect of flow velocity}

Tests were carried out at different upstream pressures between 10 bar and 40 bar, corresponding to flow velocities between 45 and $90 \mathrm{~m} / \mathrm{s}$. As discussed in Section 2.1, pitting test results depend upon only two parameters, namely the characteristic diameter $\delta$ and the coverage time $\tau$. The effect of flow velocity on both parameters and, as a consequence, on the whole distribution function of pitting rate is investigated in the present section.

Fig. 15 presents the effect of flow velocity on the pit characteristic size $\delta$. Let us recall that this characteristic size measures the pit diameter which contributes the most to the total area of deformation (see Section 3.2). It appears that pit size increases with flow velocity with a power smaller than 1 . The increase is similar for all three materials and the exponent is estimated at about 0.75 .

As for the coverage time $\tau$, Fig. 16 shows that it decreases very rapidly with the flow velocity. For all three materials, the coverage time exhibits a decrease with a power estimated at about 6.5.

Both parameters are combined in the unique parameter $1 /\left(\delta^{2} \tau\right)$ which has the same unit as the pitting rate. This parameter is the reference pitting rate to be used for making the actual pitting rate non-dimensional (see Eq. (10)). In other words, it is the scaling parameter which allows the estimate of the actual pitting rate from the non-dimensional pitting rate given by Eq. (12).

Fig. 17 shows that this reference pitting rate increases roughly like $V^{5}$ without any significant difference for the three materials 


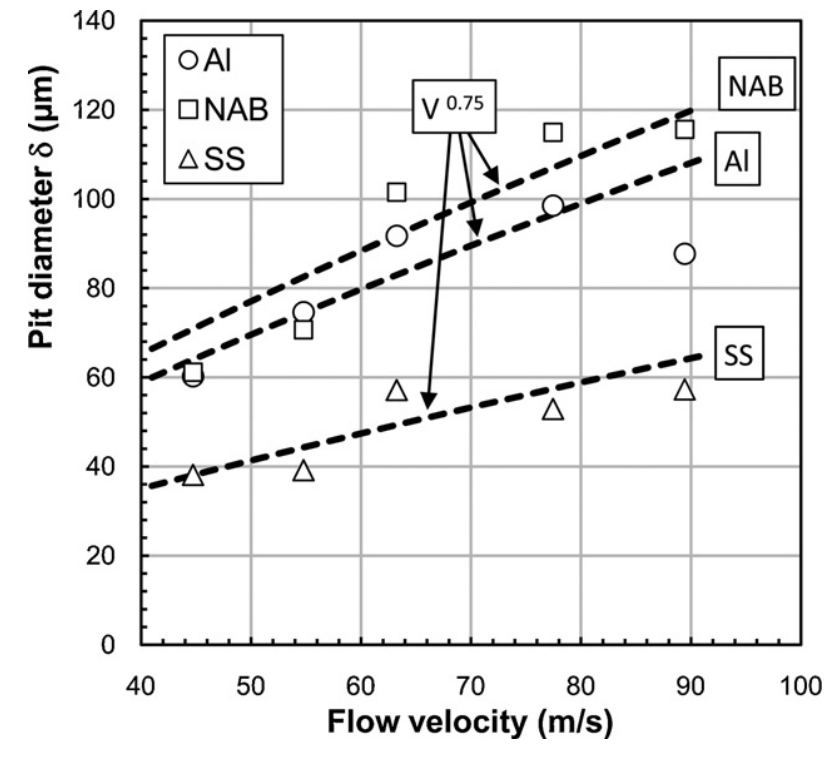

Fig. 15. Influence of flow velocity on the pit characteristic diameter $\delta$.

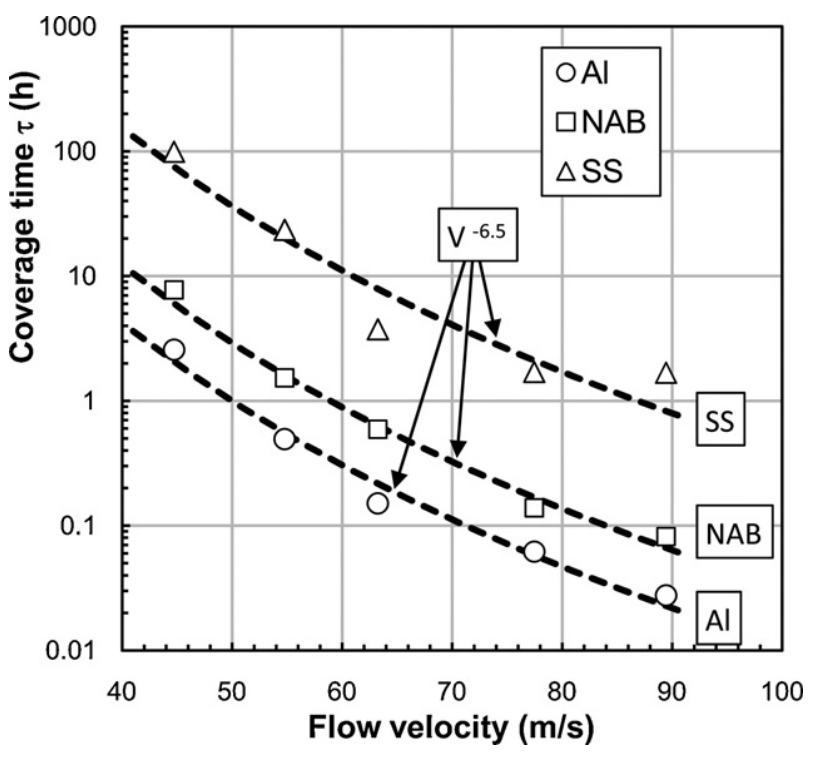

Fig. 16. Influence of flow velocity on the coverage time $\tau$.

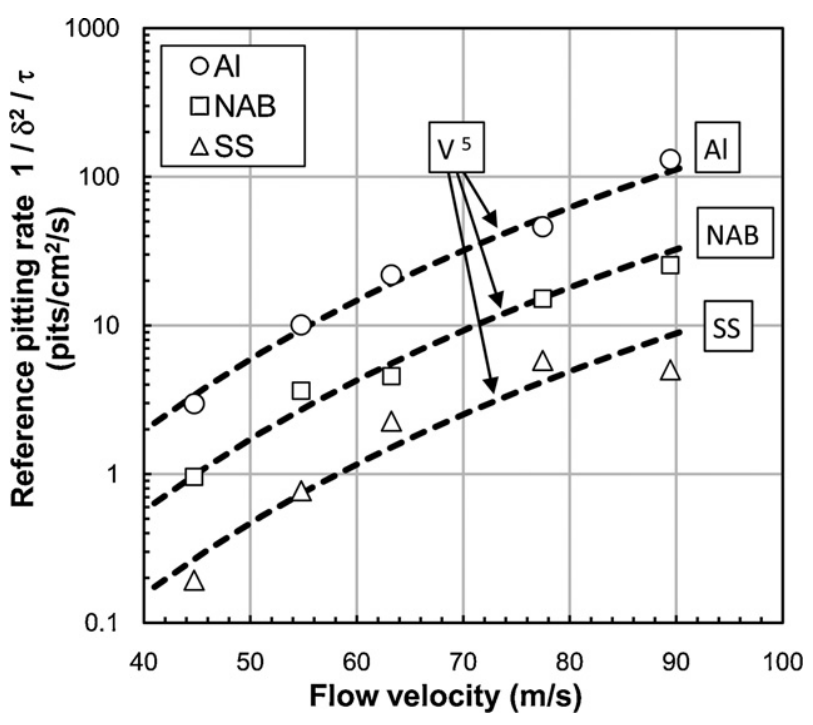

Fig. 17. Influence of flow velocity on the reference pitting rate defined by $1 / \delta^{2} \tau$. tested here. This dependency is in agreement with trends generally reported in the literature. As an example, Stinebring et al. [38] observed that the pitting rate on gives constructed of pure annealed aluminum and tested in a high-speed water tunnel follows a sixth-power law curve in a range of velocities between 14.9 and $59.3 \mathrm{~m} / \mathrm{s}$.

\section{Depth and volume of pits}

The volume of pits is correlated to the equivalent diameter of the pit plane surface area as demonstrated by Fig. 18. Let us recall that pit volume is calculated using a fully 3D approach and that no assumption is made on the shape of the pits which, in particular, are not assumed axisymmetric for volume computation. Each point in Fig. 18 represents one pit and a total number of 1258 pits are plotted corresponding to various values of the upstream pressure. This figure shows that the volume of pits follows a power law with the equivalent diameter which does not depend significantly upon the operating conditions in the present range of investigation. The exponent is slightly larger than 2 and is estimated close to 2.1 so that pit volume $v$ appears to vary like $v \propto D^{2.1}$

If it is assumed that pit shapes are geometrically similar on the average whatever may be their size, pit volume should vary like $v \propto h D^{2}$. Then, pit depth should vary like $h \propto v D^{-2} \propto D^{0.1}$ and relative pit depth should vary like $h / D \propto D^{-0.9}$. Fig. 19 confirms this behavior and consequently validates the assumption of geometric scaling for pit shape. It is important to note that the geometric similarity of pit shapes applies only to the average. Fig. 20 clearly shows that there is not a unique pit shape but a large variety of shapes since depth can vary significantly for a given equivalent diameter. The similarity law on pit shape is then valid only from a statistical viewpoint for the average. Significant deviations can appear around the average shape.

Note that the same pits have been considered in the plots of Fig. 18 for the volume, in Fig. 19 for the relative depth (ratio depth to diameter) and in Fig. 20 for the depth. The apparent difference in scattering is due to the fact that volume varies over four decades whereas depth varies over one decade only.

In order to check the influence of the cut-off depth, several analysis were conducted with different cut-off depths between 0.3 and $0.7 \mu \mathrm{m}$. Fig. 21 shows that a decrease in cut-off depth essentially leads to a translation of the curves towards larger diameters but

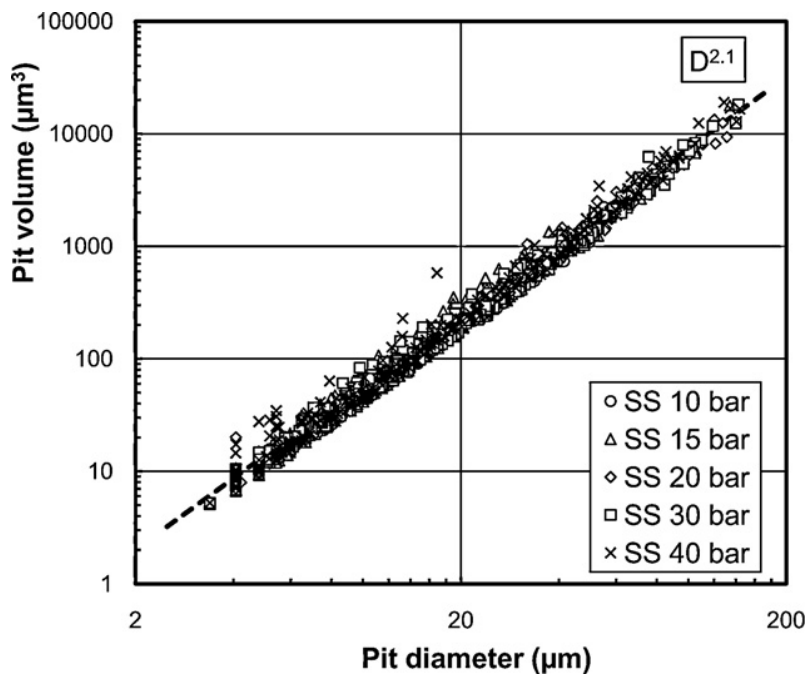

Fig. 18. Pit volume as a function of pit diameter for different values of the upstream pressure and a constant cavitation number $\sigma=0.9$ on Duplex Stainless Steel 2205 (cut-off depth: $0.5 \mu \mathrm{m}$ ). The solid line corresponds to the equation $v=0.47 D^{2.1}$ where $v$ is pit volume in $\mu \mathrm{m}^{3}$ and $D$ pit diameter in $\mu \mathrm{m}$. 


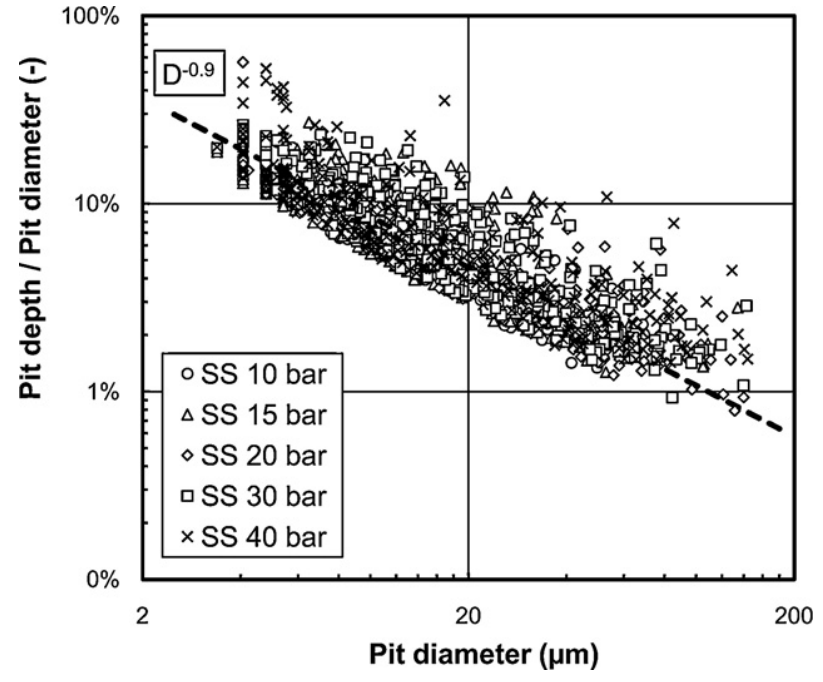

Fig. 19. Relative pit depth as a function of pit diameter for different values of the upstream pressure and a constant cavitation number $\sigma=0.9$ on Duplex Stainless Steel 2205 (cut-off depth: $0.5 \mu \mathrm{m}$ ).

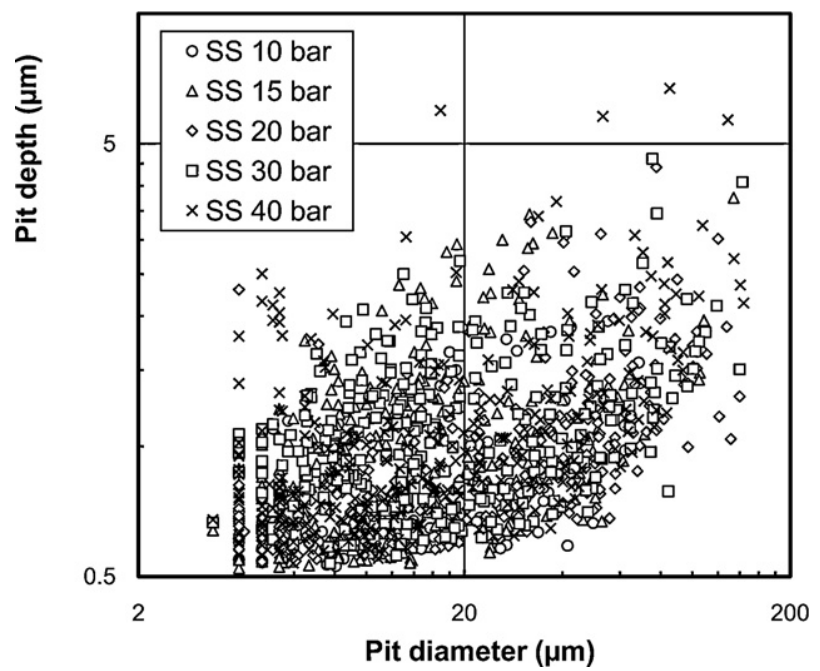

Fig. 20. Pit depth as a function of pit diameter for different values of the upstream pressure and a constant cavitation number $\sigma=0.9$ on Duplex Stainless Steel 2205. The cut-off depth of $0.5 \mu \mathrm{m}$ corresponds to the lower limit of the figure.

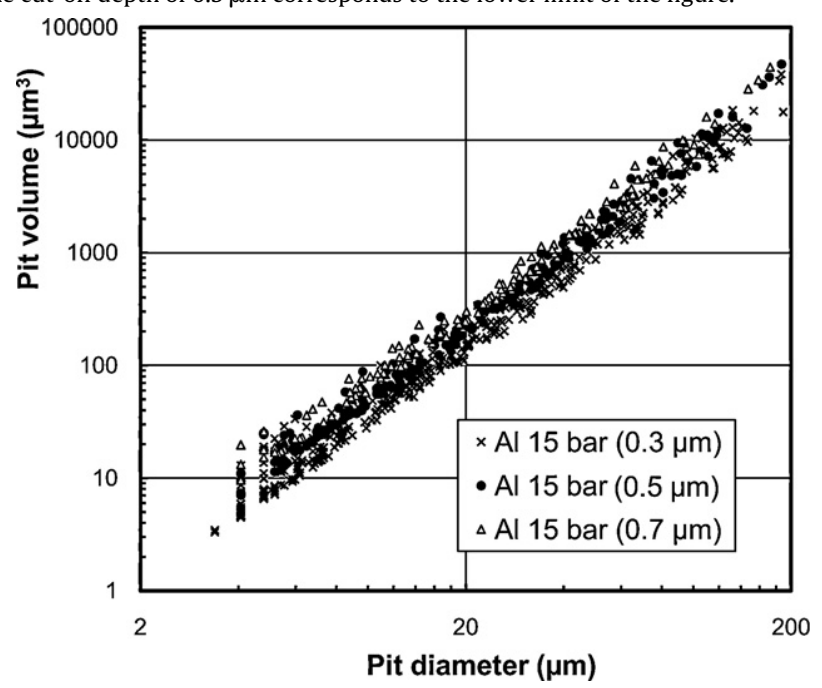

Fig. 21. Influence of cut-off depth on the relationship between pit volume and pit diameter (Aluminum alloy $\mathrm{Al} \mathrm{7075,} \mathrm{upstream} \mathrm{pressure} 15$ bar, constant cavitation number $\sigma=0.9$ ).

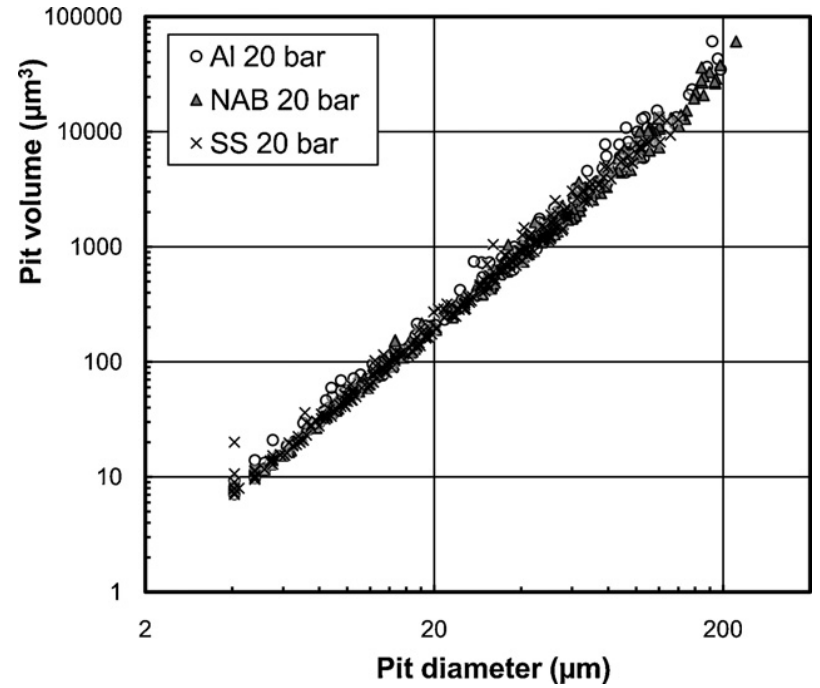

Fig. 22. Pit volume as a function of pit diameter for Aluminum Al 7075, Nickel Aluminum Bronze and Stainless Steel SS 2205 (upstream pressure 20 bars, constant cavitation number $\sigma=0.9$, cut-off depth: $0.5 \mu \mathrm{m}$ ).

the different curves remain parallel. It is then concluded that the previous trend on the evolution of pit volume with pit diameter is independent of cut-off depth.

Moreover, this trend is the same for the three tested materials (Aluminum Al 7075, Nickel Aluminum Bronze and Stainless Steel SS2205) as shown in Fig. 22.

\section{Conclusion}

The present work shows that the distribution of pits with diameter can be approximated by an exponential law. This law depends upon two parameters. One is the characteristic diameter $\delta$ of the pits which contribute mostly to the total area of erosion damage. Another is the coverage time $\tau$ which is the time required for the surface to be entirely covered just one time by the erosion pits. For each pitting test, the distribution of pits with diameter is fully described by these two parameters.

Scaling laws for pitting tests can be analyzed on the basis of both parameters. Pit size is scaled like $\delta$ and pitting rate like $\delta^{-2} \tau^{-1}$. Using these two scaling laws, a non-dimensional distribution of pits can be obtained which appears to be universal for the cavitation configuration and geometry under consideration here and in particular independent of material and operating conditions. These scaling laws were derived from pitting tests conducted on three materials, at different flow velocities in the range $45-90 \mathrm{~m} / \mathrm{s}$ and at equal cavitation number.

The characteristic pit equivalent diameter $\delta$ depends upon the material. Comparison of nano-indentation tests conducted at equal loading appears to give a good qualitative prediction of the effect of the material on pit size.

On all three materials, pit equivalent diameter increases slightly with flow velocity, approximately like $V^{0.75}$. As for the coverage time $\tau$, it strongly decreases with the flow velocity with an exponent of the order of -6.5 . This effect is probably due to both an increase in the frequency of bubbles collapsing and an increase of the pressure pulse heights with flow velocity, whereas the threshold for pitting can reasonably be expected as almost constant and only material dependent. Both reasons concur to make the coverage time strongly increase with flow velocity at constant cavitation number. As for the scale factor on pitting rate $\delta^{-2} \tau^{-1}$, present pitting tests show that it increases with the fifth power of the flow velocity, which confirms the commonly accepted strong influence of the flow velocity on pitting damage. 
The present work shows that the erosion damage (permanent deformation) during the incubation period and in particular the pitting rate is not correlated in a simple way to the elastic limit of the materials determined from conventional quasi-steady tensile tests. Further investigations are needed to clarify this issue. In particular, it would be necessary to better quantify the strain rate effect and try to correlate pitting test results with material properties at high strain rates more representative of the actual loading conditions during the cavitation erosion process.

\section{Acknowledgements}

This research was conducted under a NICOP project funded by the Office of Naval Research. The authors wish to thank Dr. KiHan Kim from the Office of Naval Research (ONR) and Dr. Richard Vogelsong from the Office of Naval Research Global (ONRG) who supported this work. They are also very grateful to Dr. Farrel Martin (Naval Research Laboratory) and Dr. Martin Donnelly (NSWCCD) for fruitful discussions. The authors would like to thank Dr. Michel Suery and Mr. Charles Josserond (from SIMAP, the Materials and Processes Science and Engineering Laboratory of the Grenoble Institute of Technology) for offering us the opportunity to complete the tensile tests.

\section{References}

[1] L. Rayleigh, On the pressure developed in a liquid during the collapse of a spherical cavity, Philosophical Magazine 34 (1917) 94-98 (Series 4).

[2] R.T. Knapp, Recent investigations of the mechanics of cavitation and cavitation damage, Transactions of the ASME (October) (1955) 1045-1054.

[3] R.T. Knapp, Accelerated field tests of cavitation intensity, Transactions of the ASME (January) (1958) 91-102.

[4] R. Fortes Patella, J.-L. Reboud, A. Archer, Cavitation damage measurement by 3D laser profilometry, Wear 246 (1-2) (2000) 59-67.

[5] M. Dular, B. Bachert, B. Stoffel, B. Sirok, Relationship between cavitation structures and cavitation damage, Wear 257 (11) (2004) 1176-1184.

[6] T. Okada, Y. Iwai, K. Awazu, A study of cavitation bubble collapse pressures and erosion. Part 1: A method for measurement of collapse pressures, Wear 133 (1989) 219-232.

[7] A. Abouel-Kasem, A.E. El-Deen, K.M. Emara, S.M. Ahmed, Investigation into cavitation erosion pits, Journal of Tribology 131 (3) (2009) 031605.

[8] M. Dular, A. Osterman, Pit clustering in cavitation erosion, Wear 265 (5-6) (2008) 811-820

[9] Y. Tomita, T. Inaba, R. Uchikoshi, T. Kodama, Peeling off effect and damage pit formation by ultrasonic cavitation, in: The International Conference on Hydraulic Machinery and Equipments, Timisoara, Romania, 2008, pp. 19-24.

[10] C. Haosheng, L. Shihan, Inelastic damages by stress wave on steel surface at the incubation stage of vibration cavitation erosion, Wear 266 (1-2)(2009) 69-75.

[11] C. Haosheng, W. Jiadao, C. Darong, Cavitation damages on solid surfaces in suspensions containing spherical and irregular microparticles, Wear 266 (1-2) (2009) 345-348

[12] C. Haosheng, L. Jiang, C. Darong, W. Jiadao, Damages on steel surface at the incubation stage of the vibration cavitation erosion in water, Wear 265 (5-6) (2008) 692-698.

[13] A. Karimi, Cavitation erosion of a Duplex Stainless Steel, Materials Science and Engineering 86 (1987) 191-203.
[14] M.A. Dominguez-Cortazar, J.P. Franc, J.M. Michel, The erosive axial collapse of a cavitating vortex: an experimental study, Journal of Fluids Engineering 119 (3) (1997) 686-691.

[15] M. Futakawa, H. Kogawa, R. Hino, H. Date, H. Takeishi, Erosion damage on solid boundaries in contact with liquid metals by impulsive pressure injection, International Journal of Impact Engineering 28 (2) (2003) 123-135.

[16] M. Futakawa, T. Naoe, H. Kogawa, C.-C. Tsai, Y. Ikeda, Pitting damage formation up to over 10 million cycles off-line test by MIMTM, Journal of Nuclear Science and Technology 40 (11) (2003) 895-904.

[17] C. Haosheng, L. Yongjian, C. Darong, W. Jiadao, Experimental and numerical investigations on development of cavitation erosion pits on solid surface, Tribology Letters 26 (2) (2007) 153-159.

[18] C. Haosheng, Iridescent rings around cavitation erosion pits on surface of mild carbon steel, Wear 269 (7-8) (2010) 602-606.

[19] T. Momma, A. Lichtarowicz, A study of pressures and erosion produced by collapsing cavitation, Wear 186-187 (Part 2) (1995) 425-436.

[20] G.L. Chahine, P. Courbière, Noise and erosion of self-resonating cavitating jets, Journal of Fluids Engineering 109 (4) (1987) 429-435.

[21] G.L. Chahine, P. Genoux, Simulation of the pressure field due to a submerged oscillating jet impacting on a solid wall, Journal of Fluids Engineering 106 (1984) 491-496.

[22] G.L. Chahine, V.E. Johnson, Mechanics and applications of self-resonating cavitating jets, in: International Symposium on Jets and Cavities, Miami, Florida, ASME, WAM, 1985.

[23] Y. Tomita, A. Shima, Mechanisms of impulsive pressure generation and damage pit formation by bubble collapse, Journal of Fluid Mechanics 169 (1986) 535-564.

[24] A. Philipp, W. Lauterborn, Cavitation erosion by single laser-produced bubbles, Journal of Fluid Mechanics 361 (1998) 75-116.

[25] J.-P. Franc, Incubation time and cavitation erosion rate of work-hardening materials, Journal of Fluids Engineering 131 (2) (2009) 021303.

[26] J. Choung, S. Cho, Study on true stress correction from tensile tests, Journal of Mechanical Science and Technology 22 (6) (2008) 1039-1051.

[27] Y. Ling, Uniaxial true stress-strain after necking, AMP Journal of Technology 5 (1996) 37-48.

[28] G.E. Dieter, Mechanical behavior of materials under tension, in: Mechanical Metallurgy, McGraw-Hill, New York, 1976, pp. 329-348.

[29] G. La Rosa, G. Mirone, A. Risitano, Postnecking elastoplastic characterization: degree of approximation in the Bridgman method and properties of the flow-stress/true-stress ratio, Metallurgical and Materials Transactions A 34 (3) (2003) 615-624.

[30] J. Lemaitre, J.L. Chaboche, Mécanique des matériaux solides, Dunod, 1996.

[31] R.W. Armstrong, S.M. Walley, High strain rate properties of metals and alloys, International Materials Reviews A 53 (3) (2008) 105-128.

[32] C. Pieralli, G. Tribillon, Traitement d'images 3D appliqué à la profilométrie optique pour l'étude du phénomène d'érosion de cavitation, Journal of Optics 18 (1) (1987) 9-18 (Paris).

[33] B. Belahadji, J.P. Franc, J.M. Michel, A statistical analysis of cavitation erosion pits, Journal of Fluids Engineering 113 (4) (1991) 700-706.

[34] S.M. Ahmed, K. Hokkirigawa, Y. Ito, R. Oba, Scanning electron microscopy observation on the incubation period of vibratory cavitation erosion, Wear 142 (1991) 303-314

[35] H. Soyama, M. Futakawa, K. Homma, Estimation of pitting damage induced by cavitation impacts, Journal of Nuclear Materials $343(1-3)$ (2005) 116-122.

[36] J.P. Franc, J.M. Michel, Fundamentals of cavitation, in: R. Moreau (Ed.), Fluid Mechanics and its Applications, Springer, 2004.

[37] R. Rodríguez-Baracaldo, J. Benito, J. Cabrera, Tensile and compressive test in nanocrystalline and ultrafine carbon steel, Journal of Materials Science 45 (17) (2010) 4796-4804.

[38] D.R. Stinebring, J.W. Holl, R.E.A. Arndt, Two aspects of cavitation damage in the incubation zone: scaling by energy considerations and leading edge damage, Journal of Fluids Engineering 102 (December) (1980) 481-485. 